\title{
Documenting correct assessment of biliary anatomy during laparoscopic cholecystectomy
}

\author{
K. T. Buddingh $\cdot$ A. N. Morks $\cdot$ H. O. ten Cate Hoedemaker • \\ C. B. Blaauw - G. M. van Dam • R. J. Ploeg • \\ H. S. Hofker • V. B. Nieuwenhuijs
}

Received: 10 November 2010/Accepted: 13 June 2011/Published online: 27 July 2011

(C) The Author(s) 2011. This article is published with open access at Springerlink.com

\begin{abstract}
Background Correct assessment of biliary anatomy can be documented by photographs showing the "critical view of safety" (CVS) but also by intraoperative cholangiography (IOC).

Methods Photographs of the CVS and IOC images for 63 patients were presented to three expert observers in a random and blinded fashion. The observers answered questions pertaining to whether the biliary anatomy had been conclusively documented.

Results The CVS photographs were judged to be "conclusive" in $27 \%$, "probable" in $35 \%$, and "inconclusive" in $38 \%$ of the cases. The IOC images performed better and were judged to be "conclusive" in 57\%, "probable" in $25 \%$, and "inconclusive" in $18 \%$ of the cases $(P<0.001$ compared with the photographs). The observers indicated that they would feel comfortable transecting the cystic duct based on the CVS photographs in 52\% of the cases and based on the IOC images in $73 \%$ of the cases $(P=0.004)$. The interobserver agreement was moderate for both methods (kappa values, 0.4-0.5). For patients with a history of cholecystitis, both the CVS photographs and the IOC images were less frequently judged to be sufficient for
\end{abstract}

K. T. Buddingh - A. N. Morks - H. O. ten Cate Hoedemaker

G. M. van Dam · R. J. Ploeg · H. S. Hofker ·

V. B. Nieuwenhuijs $(\square)$

Department of Surgery, Division of Abdominal Surgery,

University Medical Center Groningen, Postbox 30.001,

9700 RB Groningen, The Netherlands

e-mail: v.b.nieuwenhuijs@chir.umcg.nl

\section{B. Blaauw}

Department of Medical Humanities, Faculty of Medical

Sciences, Division of Medical Law, University of Groningen,

Groningen, The Netherlands transection of the cystic duct $(P=0.006$ and 0.017 , respectively).

Conclusion In this series, IOC was superior to photographs of the CVS for documentation of the biliary anatomy during laparoscopic cholecystectomy. However, both methods were judged to be conclusive only for a limited proportion of patients, especially in the case of cholecystitis. This study highlights that documenting assessment of the biliary anatomy is not as straightforward as it seems and that protocols are necessary, especially if the images may be used for medicolegal purposes. Documentation of the biliary anatomy should be addressed during training courses for laparoscopic surgery.

Keywords Bile duct injury - Cholecystectomy · Critical view of safety $\cdot$ Intraoperative cholangiography

Bile duct injury (BDI) is a dreaded complication during both laparoscopic and classic open cholecystectomy. The most severe type of BDI is complete transection of the common bile duct (CBD), and it occurs when the CBD is mistaken for the cystic duct. An important safety measure for identifying the cystic duct is to establish the so-called critical view of safety (CVS) [1]. In addition, intraoperative cholangiography (IOC) may be used to assess the biliary anatomy. Although opinions vary on whether IOC should be performed routinely or selectively, IOC is associated with a reduction in the incidence of major BDI [2-5].

Currently, the guidelines of the Dutch Society of Surgery advise that the CVS is to be documented for educational and medicolegal purposes [6]. It should be documented in the operation notes and preferably supported by intraoperative images. Two Dutch studies that assessed whether photographs or video images performed 
better at documenting the CVS yielded contradictory results [7, 8]. In both studies, the CVS (and thus, cystic duct identification) could be registered conclusively for only $34-70 \%$ of the patients.

Besides photographs of the CVS, stored IOC images can be used as documentation for correct identification of the cystic duct. Actually, IOC may constitute a better documentation than photographs of the CVS, but this has never been assessed.

In our University Medical Centre, the CVS is documented routinely by photographs, and IOC is performed routinely. In this study, we investigated the quality of the documentation of biliary anatomy using both photography of the CVS and IOC.

\section{Methods}

In the University Medical Centre Groningen, most cholecystectomies are performed by surgical trainees under the supervision of one among seven consultant surgeons specialized in gastrointestinal surgery. The standard operative technique for laparoscopic cholecystectomy at our center is the CVS technique described by Strasberg et al. [1] followed by routine IOC [9]. Digital registration of the CVS by means of photography has been hospital policy since November 2008.

\section{Patients}

All cholecystectomies between November 2008 and April 2010 were retrospectively reviewed. Patients were included in the analysis whenever they had undergone a cholecystectomy completed laparoscopically, whenever achievement of the CVS was documented in the operation notes, whenever photographs of the CVS were stored in the digital medical records, and whenever IOC had been successfully performed and saved in the digital medical file.

Review of the images

The photographs of the CVS in tagged image file format (.tiff) and the IOCs in joint photographic expert group format (.jpg) were reviewed and rated by three expert abdominal surgeons (H.O.C.H., R.J.P., and V.B.N.). Each surgeon had supervised more than 100 laparoscopic cholecystectomies after completion of surgical training.

The photographs and the IOC images were presented in random unmatched order without additional patient information. The surgeons answered consecutive questions pertaining to the quality of the images and the documentation of biliary anatomy assessment. The quality of the images was rated on a 10-point scale ranging from 1 (very poor) to 10 (excellent). The translated version of the scoring form is included as Appendix.

Statistical analysis

Statistical analysis was performed with SPPS 16.0 for Windows (SPPS Inc, Chicago, IL, USA). For analysis of continuous variables, the mean of the three observers was used. For analysis of ordinal and nominal values, the median of the three observers was used.

The paired samples $t$-test was used to compare continuous variables. The Wilcoxon paired samples signed ranks test and the McNemar test were used to compare paired ordinal and nominal variables. The Mann-Whitney $U$ test and the chi-squared test were used to compare unpaired ordinal and nominal variables. Interobserver agreement was assessed by calculating the kappa values. A $P$ value less than 0.05 was considered significant.

\section{Results}

Patients

The CVS was explicitly reported to have been achieved in 130 of 139 laparoscopically completed cholecystectomies. It was recorded by photograph for 81 patients. For the remaining 49 patients, either no images $(n=35)$ or only videos $(n=14)$ of the CVS were stored. Attempted IOC for 116 patients was successful for 97 patients. For 63 patients, both photographs of the CVS and IOC images were available. The median number of photographs taken was two (interquartile range [IQR], 1-3). The median number of IOC images stored, usually as a series in a short film, was six (IQR, 5-12).

The indications for cholecystectomy were uncomplicated gallstone disease in 31 cases (49\%), biliary pancreatitis or CBD stones in 13 cases $(21 \%)$, and current or previous cholecystitis in 19 cases $(30 \%)$. No bile duct injuries or bile leaks occurred in this series of patients.

\section{Photography versus IOC}

Table 1 shows the experts' ratings of the photographs and the IOC images. The quality of the photographs was rated lower $(5.8 \pm 1.4)$ than that of the IOCs $(6.7 \pm 1.3$; $P<0.001)$. The CVS was documented "conclusively" by photography for only 17 patients $(27 \%)$. It was rated as "probably" for 22 patients (35\%) and as "inconclusive" for 24 patients (38\%). In comparison, IOC was judged to document the cystic duct "conclusively" for 36 patients (57\%), "probably" for 16 patients (25\%), and "inconclusively" for 11 patients (18\%). Thus, IOC was superior to 
Table 1 Registration of the cystic duct by photograph and intraoperative cholangiography (IOC)

\begin{tabular}{llll}
\hline & Photograph & IOC & $P$ value \\
\hline $\begin{array}{l}\text { Mean quality of the images } \\
\text { CVS/cystic duct }\end{array}$ & $5.8 \pm 1.4$ & $6.7 \pm 1.3$ & $<0.001^{\mathrm{a}}$ \\
$\quad$ identified: $n(\%)$ & & $<0.001^{\mathrm{a}}$ \\
$\quad$ Yes & $17(27.0)$ & $36(57.1)$ & \\
Probably & $22(34.9)$ & $16(25.4)$ & \\
$\quad$ Inconclusive & $24(38.1)$ & $11(17.5)$ & \\
Safe to transect duct: $n(\%)$ & & & $0.004^{\mathrm{a}}$ \\
Yes & $33(52.4)$ & $46(73.0)$ & \\
No & $30(47.6)$ & $17(27.0)$ & \\
\hline
\end{tabular}

$C V S$ critical view of safety

${ }^{\text {a }}$ Indicates $P<0.05$

photography of the CVS for documenting assessment of the biliary anatomy $(P<0.001)$.

Based on the photographs, the surgeons deemed it justifiable to transect the cystic duct in 33 patients $(52 \%)$. Based on the IOC, transection of the cystic duct was deemed justifiable for 46 patients $(73 \% ; P=0.004)$. Correct documentation of the biliary anatomy was not associated with a higher number of photographs stored $(P=0.747)$ nor with a higher number of IOC images stored $(P=0.950)$.

For 14 patients, neither of the two methods was judged conclusive. Review of the operative notes for these patients showed expression of doubts about the way the CVS was visualized for 4 of the 14 patients and doubts about the IOC for 3 of the 14 patients. Figure 1 portrays a conclusively documented CVS in three photographs. Figure 2 shows a case of correctly and a case of incorrectly documented IOC.

The inflamed gallbladder

Table 2 shows the differences between patients who underwent cholecystectomy for current or previous cholecystitis and those with other indications. For the 19 patients with a history of cholecystitis, the surgeons deemed the photographs of CVS sufficiently conclusive to transect the cystic duct in five patients $(26 \%)$. Among the patients undergoing cholecystectomy for other indications, this was $28(64 \%)$ of 44 patients $(P=0.004)$.

The same phenomenon was seen for IOC. Transsection was deemed safe for $53 \%$ of the patients with past or current cholecystitis versus $82 \%$ of those with other indications $(P=0.017)$. The results are shown in Table 2.

Interobserver agreement

The kappa for interobserver agreement on conclusiveness of photographs of the CVS was 0.416 between observers A and B, 0.499 between observers A and C, and 0.394 between observers $\mathrm{B}$ and $\mathrm{C}$. The kappas for interobserver agreement on conclusiveness of the IOCs were 0.533 , 0.478 , and 0.407 , respectively. These values have been described to indicate moderate agreement [10].

\section{Discussion}

This study investigated the documentation of correct assessment of biliary anatomy by photography of the CVS and by IOC. The cystic duct was conclusively documented in $57 \%$ of the IOCs compared with $27 \%$ of the photographs of the CVS. Conclusive documentation of the biliary anatomy was especially poor for patients with a history of cholecystitis.

Several studies have previously evaluated photographs of the CVS $[7,8,11]$. The rate of conclusive photographs in the current study was lower than in the other studies. This difference may be explained partly by the high proportion of patients with cholecystitis in the current study (Table 3). In a recent commentary, Strasberg and Brunt [12] describe the achievement of CVS as more challenging with an inflamed gallbladder. Due to the altered aspect of the anatomic structures during or after inflammation, it may be especially difficult to capture the CVS in one or two still images. Doubts were expressed about the CVS or IOC in the operative notes for only a minority of patients with inconclusive documentation in photographs or IOC. Therefore, the problem probably lies in the documentation rather than in unsafely performed surgery. Nonetheless, the proportion of properly documented CVS is unacceptably low, and effort currently is being put into improving this aspect of gallbladder surgery at our center. New protocols including video images and instructions during resident courses in laparoscopic surgery have been implemented for this purpose.

The CVS technique is fully accepted in Dutch surgical practice. A nationwide survey by our group showed that $98 \%$ of the surgeons apply this technique [13]. Also, many surgeons document the CVS by photograph (43\%) or video (30\%). Considering the poor results from photography of the CVS at our center, it would be interesting to assess the quality of the images from other hospitals.

Previous studies have assessed whether the CVS had been achieved "certainly," "probably," or "inconclusively" [7, 8]. In the current study, a binary response ("yes" or "no") also was elicited from the observers by asking them whether they would feel comfortable transecting the identified duct based on the images. Half of the responses marked as "probably" then changed to "yes." The other half changed to "no." This illustrates the range of responses that may be classified as "probably." The 

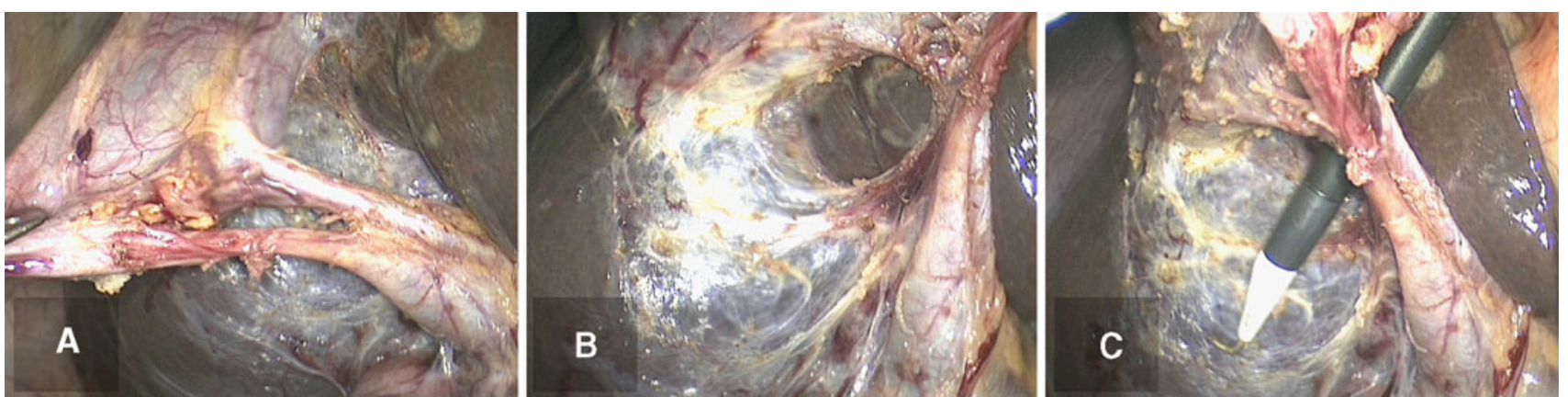

Fig. 1 Correct documentation of the critical view of safety in three photographs. A Medial view. B Lateral view. C View with an instrument through one of the windows to enhance depth perception

Fig. 2 Documentation of the biliary anatomy by intraoperative cholangiography. A Performed correctly. The trajectory of the cystic duct is clearly visible, as well as the intrahepatic bile ducts, the common bile duct, and the duodenum. B Performed incorrectly. Although the intrahepatic ducts and the duodenum are visualized, the cystic duct is not, and it could be the common bile duct that is cannulated instead of the cystic duct

Table 2 Performance of photography and intraoperative cholangiography (IOC) in patients with and without cholecystitis
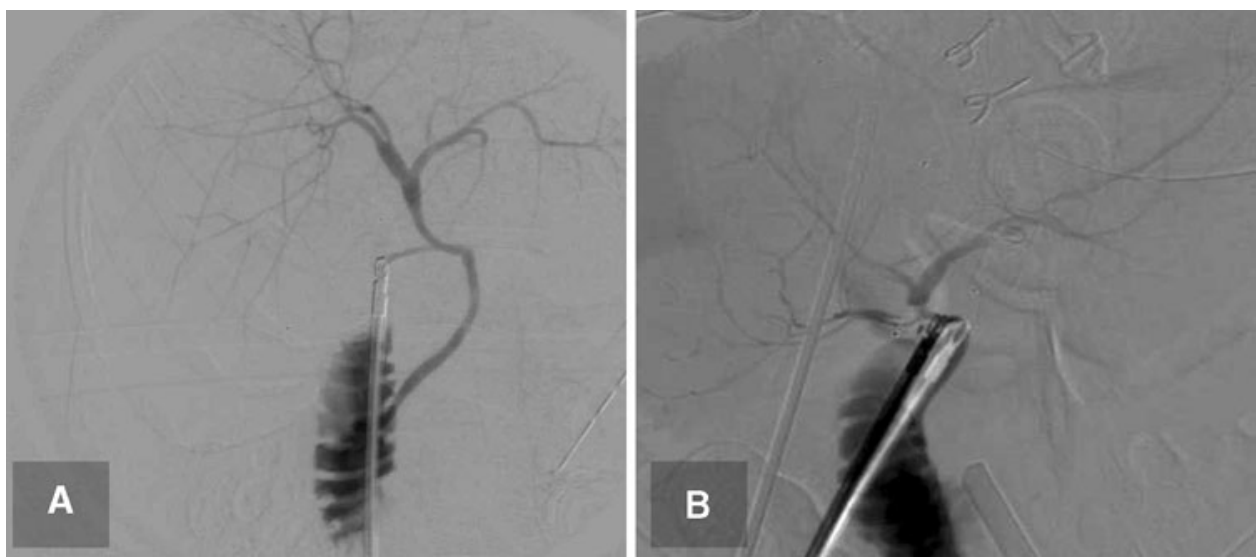

\begin{tabular}{llll}
\hline & $\begin{array}{l}\text { Current or previous } \\
\text { cholecystitis }(n=19)\end{array}$ & $\begin{array}{l}\text { Other indications } \\
(n=44)\end{array}$ & $P$ value \\
\hline Quality photo & $5.5 \pm 1.4$ & $6.0 \pm 1.4$ & 0.212 \\
Quality IOC & $6.5 \pm 1.3$ & $6.8 \pm 1.3$ & 0.351 \\
CVS on photograph: $n(\%)$ & $2(10.5)$ & $15(34.1)$ & $0.007^{\mathrm{a}}$ \\
Yes & $5(26.3)$ & $17(38.6)$ & \\
Probably & $12(63.2)$ & $12(27.3)$ & $0.006^{\mathrm{a}}$ \\
Inconclusive & & $28(63.6)$ & \\
Transect duct based on photo: $n(\%)$ & $5(26.3)$ & $16(36.4)$ & 0.099 \\
Yes & $14(73.7)$ & $27(61.4)$ & \\
No & & $13(29.5)$ & \\
Cystic duct identified by IOC: $n(\%)$ & $9(47.4)$ & $4(9.1)$ & \\
Yes & $3(15.8)$ & $36(81.8)$ & \\
Probably & $7(36.8)$ & $8(18.2)$ & \\
Inconclusive & & & \\
Transect duct based on IOC: $n(\%)$ & $10(52.6)$ & $9(47.4)$ & \\
Yes & & & \\
No & & & \\
\hline
\end{tabular}

${ }^{\text {a }}$ Indicates $P<0.05$

interobserver agreement on the photographs was moderate, with kappa values between 0.4 and 0.6 . The only previous study to assess interobserver agreement on CVS photographs found a slightly higher kappa of 0.69 (fair agreement) [7]. This study cannot with certainty explain the low interrater agreement, but we believe it would benefit from higher-quality photographs according to a standardized protocol.

The merits of IOC have been described in large population-based studies [2-4]. There is, however, concern that 
Table 3 Documentation of critical view of safety (CVS) by photograph in previous studies and in the current study

\begin{tabular}{|c|c|c|c|c|c|}
\hline & $\begin{array}{l}\text { Rawlings et al. [11] } \\
\text { (single port) }(\%)\end{array}$ & $\begin{array}{l}\text { Plaisier et al. } \\
{[8](\%)}\end{array}$ & $\begin{array}{l}\text { Emous et al. [7] (mean } \\
\text { of two observers) }(\%)\end{array}$ & $\begin{array}{l}\text { Current study } \\
\text { (photo CVS) (\%) }\end{array}$ & $\begin{array}{l}\text { Current study } \\
\text { (IOC) }(\%)\end{array}$ \\
\hline \multicolumn{6}{|l|}{ CVS/cystic duct identified } \\
\hline Yes & 64 & 62 & 40 & 27 & 57 \\
\hline Probably & 24 & 16 & 36 & 35 & 25 \\
\hline Inconclusive & 12 & 22 & 26 & 38 & 18 \\
\hline Present/previous cholecystitis & 0 & 10 & 28 & 30 & 30 \\
\hline
\end{tabular}

$I O C$ intraoperative cholangiography

IOCs are not always correctly interpreted $[14,15]$. In the current series, the cystic duct could be conclusively documented in only $57 \%$ of cases. In the cases wherein IOC did not correctly document the cystic duct, this was caused by projection of the cystic duct over the $\mathrm{CBD}$, incomplete filling of the biliary tree, or both. The interobserver agreement on the IOCs was moderate.

An unexpected finding was that a lower proportion of IOCs were conclusive for patients with an inflamed gallbladder. This may have been caused by adhesions or alterations in the morphology of the cystic duct that made the situation more prone to over projection or insufficient filling of the biliary tree during IOC.

Attention should be paid to the legal implications of documentation of the biliary anatomy. This seems evident for IOC because it is part of the radiology studies in the patient medical file. However, stored laparoscopic images, particularly images of the CVS, are relatively new items in the patient medical records. The medicolegal value of these images has not been determined. Once a selection of intraoperative images is stored, the images are considered "personal data" under Article 2 of the Dutch Personal Data Protection Act (in Dutch, abbreviated as WBP). According to this Act, special requirements regarding the quality and admissibility of data processing must be met (Article 6-15 of the Personal Data Protection Act). One of these requirements is the patient's consent for the CVS to be stored. Generally, it is accepted that the patient's consent for surgery also comprises consent for CVS documentation and storage.

Under Dutch law (Article 453 and 454 of the Medical Treatment Contracts Act, in Dutch, abbreviated as WGBO), the CVS should be documented in the patient medical records to comply with the care provider's responsibility in view of the applicable professional standard (in the case of cholecystectomy, the Dutch Guidelines and Best Practice for laparoscopic cholecystectomy [6]). The patient has certain rights in relation to his medical file (e.g., the right to access the file and to copy it) including radiology studies and laparoscopic images. The patient may use such copies in a court of law, for example, in case of bile duct injury (BDI).
On the other hand, documentation of the biliary anatomy can be used by the surgeon to substantiate measures taken to ensure safe cholecystectomy. In particular circumstances, the physician may use documents and images from the patient's file in legal procedures without the patient's consent to prove he has met requirements of due care under the professional standard. This exception is based on Article 6 of the European Convention of Human Rights, which states that everyone, including physicians, has the right of fair trial.

Besides the patient and the physician, the public prosecutor and the health care inspectorate also may claim the medical file. Dutch regulations on the quality of health care require that any calamity (an unintended adverse event resulting in the death or serious harm of a patient) in a health care institution must be reported to the Health Care Inspectorate. In case the Inspectorate encounters any violation of these regulations, the Public Prosecutor is informed.

Several studies have assessed litigation claims for iatrogenic BDI during cholecystectomy [16-20], concluding that litigation for BDI continues to play a role in modern surgical practice. Very little data exist on the role of patient safety interventions in these cases. Most of the injuries occurred before widespread implementation of the CVS technique. It would be interesting to assess claims for BDI in the years after the introduction of the CVS, especially in the Netherlands as documentation of the CVS is incorporated into the national guidelines. At the moment, documentation of the CVS in the operation notes probably is sufficient to convince a court of law that the appropriate safety measures were taken. However, it is clear that the operation notes in (gallbladder) surgery are limited in their correlation with the actual procedure [21]. As the storing of laparoscopy images becomes more widely practiced, operation notes supported by images probably will become the new standard of care.

This study was conducted retrospectively, and no protocol for taking the photographs of the CVS was used. This is, however, the first study to assess the value of IOC for documenting the cystic duct. It would be interesting to 
compare IOC with videos of the CVS in addition to photographs. Emous et al. [7] have suggested that videos of the CVS are superior to photographs, although Plaisier et al. [8] claimed that photographs are superior. Further study on this topic is currently ongoing at our center.

\section{Conclusion}

In this series, IOC was superior to photographs of the CVS for documenting the correct assessment of the biliary anatomy during laparoscopic cholecystectomy. However, both methods were conclusive only for a limited proportion of patients, especially in the case of cholecystitis. Our study highlights that documentation of the biliary anatomy is not as straightforward as it seems and that protocols are necessary, especially because the images may be used for medicolegal purposes. Documentation of cystic duct identification should be addressed during training courses for laparoscopic surgery.

Disclosures K. T. Buddingh, A. N. Morks, H. O. ten Cate Hoedemaker, C. B. Blaauw, G. M. van Dam, R. J. Ploeg, H. S. Hofker, and V. B. Nieuwenhuijs have no conflicts of interest or financial ties to disclose.

Open Access This article is distributed under the terms of the Creative Commons Attribution Noncommercial License which permits any noncommercial use, distribution, and reproduction in any medium, provided the original author(s) and source are credited.

\section{Appendix: Questions answered by each observer (translated from Dutch)}

\section{Part 1: Critical view of safety (CVS)}

1. What is the quality of the photos (grade best photo)?

(Very poor) $1-2-3-4-5-6-7-8-9-10$ (Excellent)

2. Has the CVS been achieved?

- Yes

- Probably

- Inconclusive

3. Would you transect the cystic duct based on this image?

- Yes

- No

Part 2: Intraoperative cholangiography (IOC)

1. What is the quality of the IOC (grade best image)?

(Very poor) $1-2-3-4-5-6-7-8-9-10$ (Excellent)
2. Is the duct that is cannulated the cystic duct?

- Yes

- Probably

- Inconclusive

3. Would you transect the cannulated duct based on this IOC?

- Yes

- No

\section{References}

1. Strasberg SM, Hertl M, Soper NJ (1995) An analysis of the problem of biliary injury during laparoscopic cholecystectomy. J Am Coll Surg 180:101-125

2. Fletcher DR, Hobbs MS, Tan P, Valinsky LJ, Hockey RL, Pikora TJ, Knuiman MW, Sheiner HJ, Edis A (1999) Complications of cholecystectomy: risks of the laparoscopic approach and protective effects of operative cholangiography: a population-based study. Ann Surg 229:449-457

3. Flum DR, Dellinger EP, Cheadle A, Chan L, Koepsell T (2003) Intraoperative cholangiography and risk of common bile duct injury during cholecystectomy. JAMA 289:1639-1644

4. Waage A, Nilsson M (2006) Iatrogenic bile duct injury: a population-based study of 152,776 cholecystectomies in the Swedish Inpatient Registry. Arch Surg 141:1207-1213

5. Buddingh KT, Nieuwenhuijs VB, van Buuren L, Hulscher JBF, de Jong JS, van Dam GM (2011) Intraoperative assessment of biliary anatomy for prevention of bile duct injury: a review of current and future patient safety interventions. Surg Endosc. doi: 10.1007/s00464-011-1639-8

6. Lange JF, Stassen LPS (2006) Best practice: De techniek van de laparoscopische cholecystectomie (Critical View of Safety [CVS]; Werkgroep Endoscopische Chirurgie van de Nederlandse Vereniging voor Heelkunde)

7. Emous M, Westerterp M, Wind J, Eerenberg JP, van Geloven AA (2010) Registering the critical view of safety: photo or video? Surg Endosc 24:2527-2530

8. Plaisier P, Pauwels M, Lange J (2001) Quality control in laparoscopic cholecystectomy: operation notes, video or photo print? HPB Oxford 3:197-199

9. Buddingh KT, Weersma RK, Savenije RA, van Dam GM, Nieuwenhuijs VB (2011) Lower rate of major bile duct injury and increased intraoperative management of common bile duct stones after implementation of routine intraoperative cholangiography. J Am Coll Surg [Epub ahead of print]

10. Landis JR, Koch GG (1977) The measurement of observer agreement for categorical data. Biometrics 33:159-174

11. Rawlings A, Hodgett SE, Matthews BD, Strasberg SM, Quasebarth M, Brunt LM (2010) Single-incision laparoscopic cholecystectomy: initial experience with critical view of safety dissection and routine intraoperative cholangiography. J Am Coll Surg 211:1-7

12. Strasberg SM, Brunt LM (2010) Rationale and use of the critical view of safety in laparoscopic cholecystectomy. J Am Coll Surg 211:132-138

13. Buddingh KT, Hofker HS, Cate Hoedemaker HO, van Dam GM, Ploeg RJ, Nieuwenhuijs VB (2011) Safety measures during cholecystectomy: results of a nationwide survey. World J Surg 35:1235-1241

14. Way LW, Stewart L, Gantert W, Liu K, Lee CM, Whang K, Hunter JG (2003) Causes and prevention of laparoscopic bile duct 
injuries: analysis of 252 cases from a human factors and cognitive psychology perspective. Ann Surg 237:460-469

15. Woods MS, Traverso LW, Kozarek RA, Donohue JH, Fletcher DR, Hunter JG, Oddsdottir M, Rossi RL, Tsao J, Windsor J (1995) Biliary tract complications of laparoscopic cholecystectomy are detected more frequently with routine intraoperative cholangiography. Surg Endosc 9:1076-1080

16. Alkhaffaf B, Decadt B (2010) 15 years of litigation following laparoscopic cholecystectomy in England. Ann Surg 251:682-685

17. de Reuver PR, Wind J, Cremers JE, Busch OR, van Gulik TM, Gouma DJ (2008) Litigation after laparoscopic cholecystectomy: an evaluation of the Dutch arbitration system for medical malpractice. J Am Coll Surg 206:328-334
18. Scurr JR, Brigstocke JR, Shields DA, Scurr JH (2010) Medicolegal claims following laparoscopic cholecystectomy in the UK and Ireland. Ann R Coll Surg Engl 92:286-291

19. de Reuver PR, Dijkgraaf MG, Gevers SK, Gouma DJ (2008) Poor agreement among expert witnesses in bile duct injury malpractice litigation: an expert panel survey. Ann Surg 248:815-820

20. Chandler JG, Voyles CR, Floore TL, Bartholomew LA (1997) Litigious consequences of open and laparoscopic biliary surgical mishaps. J Gastrointest Surg 1:138-145

21. Stewart L, Hunter JG, Wetter A, Chin B, Way LW (2010) Operative reports: form and function. Arch Surg 145:865-871 